\title{
How to identify pregnant women at risk of pre-eclampsia? - a review of the current literature
}

\author{
Katarzyna Kosińska-Kaczyńska, Mirosław Wielgoś \\ $1^{\text {st }}$ Department of Obstetrics and Gynecology, Medical University of Warsaw, Poland
}

\begin{abstract}
Pre-eclampsia remains a major cause of poor perinatal outcome worldwide. As administering acetylsalicylic acid in a high risk population reduces the risk of $\mathrm{PE}$, it is essential to identify women at risk of $\mathrm{PE}$. Several algorithms for $\mathrm{PE}$ risk assessment have been developed. They include maternal factors combined with uterine artery pulsatility index, mean arterial pressure, serum pregnancy-associated plasma protein-A, placental growth factor, and serum soluble fms-like tyrosine kinase-1. Beside PE prophylaxis with acetylsalicylic acid, a proper management of women considered at a high risk of PE is essential. The sFIt-1:PIGF ratio between 20 and $34+6$ weeks may be used to predict a short-term absence of PE or to predict the risk of PE diagnosis within 4 weeks and a significant shortening of the duration of pregnancy associated with it. The sFlt-1:PIGF ratio may be helpful in deciding about hospitalization or choosing the optimal time for corticosteroid administration in women at risk of PE. It may also help to reduce overall healthcare costs.
\end{abstract}

Key words: pre-eclampsia, prediction, PIGF, sFlt-1, risk factors

\section{INTRODUCTION}

Hypertension affects about $6-8 \%$ of all pregnant women [1]. In Poland the estimated number of women suffering from hypertension during pregnancy is around 30,000 per year. Although pre-eclampsia (PE) affects $2-3 \%$ of patients, it carries a great risk of maternal and perinatal mortality and morbidity [1-3]. According to the World Health Report of 2005, $12 \%$ of all maternal deaths were related to eclampsia worldwide [4]. In the USA the rate of any pre-eclampsia or mild pre-eclampsia was quite constant over the last three decades. On the other hand, over a two-fold increase in severe PE occurrence was observed during that period [5] which puts such women at the highest risk of poor perinatal outcome.

The results of the ASPRE Study were published in 2017. According to the study, administering acetylsalicylic acid (ASA) since the first trimester of pregnancy in a high-risk population reduced the occurrence of $\mathrm{PE}<34$ weeks by $80 \%$ and $<37$ weeks by $63 \%$ [6]. A recently published meta-analysis by Roberge et al. included sixteen trials on ASA in PE prevention with 18,907 participants. The main finding was that ASA reduced the risk of preterm PE when it was initiated at $\leq 16$ weeks of gestation at a daily dose of $\geq 100 \mathrm{mg}$ [7]. Nowadays ASA is widely recommended in PE prevention [8, 9]. Therefore, it is essential do correctly identify women at risk of PE to manage them with proper care in order to minimize the risks.

\section{MATERNAL RISK FACTORS OF PE}

According to the Regulation of the Minister of Health as of the 9th of November 2015 maternal risk factors of PE are:

- a history of severe PE and/or intrauterine growth restriction,

- chronic hypertension,

- chronic renal diseases,

- pre-gravid diabetes mellitus,

- body mass index (BMI) $>30$,

- autoimmune diseases (e.g. systemic lupus erythematosus),

- antiphospholipid syndrome or thrombophilia [8].

This list of risk factors is very similar to the one published by the American College of Obstetricians and Gynecologists (ACOG) in "Hypertension in Pregnancy" in 2013 [1]. ACOG 
recommends qualifying women at a high risk of PE according to the above mentioned risk factors and if they are over 40 years old, primiparous, in multiple pregnancy, after in-vitro fertilization procedure, or if their first-degree relative had a history of PE. The National Institute for Health and Care Excellence (NICE) published an overview on hypertension in pregnancy, which was last updated in 2017. According to this document two categories of risk factors of PE are distinguished. Women at a high risk of PE are those with hypertensive disease during the previous pregnancy, chronic kidney diseases, autoimmune diseases such as systemic lupus erythematosus or antiphospholipid syndrome, type 1 or type 2 diabetes or chronic hypertension. Primiparity, age $>40$ years, pregnancy interval of $>10$ years, pre-gravid $\mathrm{BMI}>35$, family history of PE and multiple pregnancy are considered to be moderate risk factors. The presence of two risk factors are considered equal to one high-risk factor [10].

Taking into account only the above mentioned maternal determinants results in the detection rate of PE of around $40 \%$. Wright et al. developed a model for the prediction of PE on the basis of maternal characteristics and medical history. 120,492 women were screened for PE between $11+0$ and $13+6$ weeks of gestation. A total of 2,704 of them experienced $P E$ in the further course of pregnancy (2.2\%). The model including maternal characteristics and history predicted $40 \%$ of all PE cases, $48 \%$ of cases of PE requiring delivery $<37$ weeks and $54 \%$ of cases of PE requiring delivery $<34$ weeks of gestation [11]. This leads to a conclusion that about $50-60 \%$ of pregnant women with PE have no possibility to have the risk factors determined in the first trimester of pregnancy. Estimating the risk of PE only on the basis of maternal and family history causes an underestimation of the number of women at risk of PE during the current pregnancy.

\section{ALGORITHMS OF PE PREDICTION}

The utility of the predictive test depends on its sensitivity, specificity but also the overall prevalence of the disease. The best way to assess the utility of the test is to analyze its likelihood ratio. Positive likelihood ratio (LR) is the proportion of patients with a condition who have positive test results to those without the condition who have identical test results. As PE is a rare condition, the screening test should have a high positive LR for adequate prediction of the probability of PE with low negative LR to exclude the disorder with confidence. A useful prediction test of $P E$ would require a positive $L R>10$ for a positive test and a negative $L R<0.2$ for a negative test [1].

A model of PE prediction based on maternal characteristics, biophysical and biochemical markers was published in 2016 by a group from King's College in London. Maternal factors were combined with uterine artery pulsatility index
(UTPI), mean arterial pressure (MAP), serum pregnancy-associated plasma protein-A (PAPP-A), and placental growth factor (PIGF) expressed as multiples of the median values. The test was applied in almost 36,000 of pregnant women between $11+0$ and $13+6$ gestational weeks, $2.9 \%$ of whom subsequently developed PE. Combining all the above mentioned factors allowed to detect $82 \%$ of $\mathrm{PE}$ requiring delivery at $<32$ weeks (95\% coefficient interval (CI) 70-90), 59\% of $P E$ requiring delivery between $32+0$ and $36+6$ weeks ( $95 \%$ $\mathrm{Cl} 52-65), 37 \%$ of $\mathrm{PE}$ requiring delivery between $37+0$ and $39+6$ weeks ( $95 \% \mathrm{Cl} 33-41)$ and $26 \%$ of $\mathrm{PE}$ requiring delivery beyond 40 weeks of gestation $(95 \% \mathrm{Cl} 2132)$ at a $5 \%$ false positive rate. At false positive rate of $10 \%$ the detection rates are $89 \%$ (79-96), 71\% (64-77), 54\% (49-58) and 38\% (32-44), respectively [12]. Different combinations of the above mentioned factors have lower detections rates. For example, taking into account maternal factors, MAP, UTPI and PAPP-A (without PIGF) allowed to detect $83 \%$ of all PE cases requiring delivery below 32 weeks of gestation $(95 \%$ $\mathrm{Cl} 72-91)$ with a $10 \%$ false positive rate [12].

Further algorithms of PE risk estimation during the second and third trimester of pregnancy have been developed. Gallo et al. presented a model of PE prediction between 19 and 24 gestational weeks. It included maternal factors, UTPI, MAP, PIGF and serum soluble fms-like tyrosine kinase-1 (sFlt-1) [2]. sFlt-1, also known as soluble vascular endothelial growth factor (VEGF) receptor 1 binds both circulating VEGF and PIGF, thus decreasing their concentration in uteroplacental and maternal circulation [3]. In PE, circulating maternal serum levels of sFlt-1 are increased, and PIGF levels are decreased $[13,14]$. sFlt-1 causes vasoconstriction and endothelial damage that may lead to PE [15]. Importantly, the increase in sFlt-1 and decrease in PIGF serum levels are ahead of the clinical symptoms of PE by 5 weeks [16]. The algorithm presented by Gallo et al. allowed to detect $52 \%, 47 \%$, and $37 \%$ of $\mathrm{PE}$ at $<32,<37$, and $\geq 37$ weeks of gestation, respectively, at a false-positive rate of $10 \%$ [2].

Similar algorithms have been developed for PE prediction at 30-34 weeks and 35-37 weeks of gestation. Predicting $98 \%(95 \% \mathrm{Cl} 88-100)$ of preterm-PE and $49 \%$ (95\% Cl 42-57) of term-PE at a false-positive rate of 5\% [17] was possible with the implementation of the same factors as in the second trimester of pregnancy. The application of the algorithm to patients at 35-37 weeks of gestation predicted PE in 82\% (95\% Cl 70-91) [18].

Basing on the above mentioned studies Fetal Medicine Foundation developed a calculator for PE risk estimations, which is available online (https://fetalmedicine.org/research/assess/preeclampsia). It allows to estimate the risk of PE using different algorithms in the first, second and third trimester of pregnancy. The questionnaire fields include all the risk factors used in the algorithm used for the specific 
interval of pregnancy weeks. However, in case of the lack of any biomarkers, the calculator will use a different algorithm to calculate the risk of $\mathrm{PE}$. The result is given with a comment qualifying it as low or high risk and with advice on further management.

\section{A SHORT-TERM PREDICTION AND EXCLUSION OF PE}

In 2016 the PROGNOSIS study on the predictive value of sFIt-1:PIGF ratio in women with suspected PE was published in the New England Journal of Medicine. A prospective multicenter observational research on 1,273 women between 24 and $36+6$ weeks of gestation with suspected PE was performed. sFIt-1:PIGF ratio of 38 or lower was predictive of the absence of PE within one week (negative predictive value $99.3 \%$, 95\% Cl 97.9-99.9), with $80 \%$ sensitivity (95\% Cl 51.9-95.7) and 78.3\% specificity (95\% Cl 74.6-81.7). The positive predictive value of sFlt-1:PIGF ratio at or above 38 for a diagnosis of PE within 4 weeks was $36.7 \%$ (95\% Cl 28.4-45.7), with $66.2 \%$ sensitivity (95\% Cl 54.0-77.0) and $83.1 \%$ specificity $(95 \% \mathrm{Cl} 79.4-86.3)$. In conclusion, the authors stated that sFlt-1:PIGF ratio of 38 or lower may be used to predict the short-term absence of PE in women in whom the syndrome is clinically suspected [19]. Moreover, sFIt-1:PIGF ratio above 38 was associated with a significantly shorter time to delivery regardless of PE status [20]. If the test was applied between 24 and $33+6$ gestational weeks and the results were $\geq 38$, the likelihood of imminent delivery was 2.9 fold greater than if the test result was $<38$. In women with sFlt-1:PIGF ratio exceeding 38 the time to delivery was also $38 \%$ shorter than in women with the test ratio of 38 or below. In 250 women with sFlt-1:PIGF ratio above 38 the mean time from the test to delivery was 17 days (interquartile range 10-26 days) [20].

Recently, a meta-analysis and a systematic review of sFIt-1:PIGF ratio in the prediction of PE has been published in Hypertension by Agrawal et al. The meta-analysis consisted of 15 studies, 8 of which included pregnant women at a high risk of $\mathrm{PE}$ and 7 at a low risk of PE (534 cases of PE and 19,587 controls). The pooled sensitivity of sFlt-1:PIGF ratio in predicting $\mathrm{PE}$ was $0.80(95 \% \mathrm{Cl} 0.68-0.88)$ and the pooled specificity was $0.92(95 \% \mathrm{Cl} 0.87-0.96)$. The positive LR was 10.5 (6.2-18) and negative LR 0.22 (0.13-0.35), which are very similar to the expected performance of a useful prediction test for PE [3]. Therefore, if the test is positive, the probability of having PE increases to $78 \%$, while if the test is negative, it decreases to $7 \%$.

The presented performance of the test makes it useful in clinical practice. It allows a more accurate prediction of the onset of PE and better patient management. sFIt-1:PIGF ratio is helpful in deciding on follow-up and hospitalization of women at risk of $\mathrm{PE}$ and in deciding on the optimal time for corticosteroid administration in case of a high risk of preterm delivery [21]. The impact on budget by implying sFlt-1:PIGF ratio as a prediction test in women with suspected $P E$ was analyzed in Italian population by Frusca et al. An economic model was developed to estimate the net financial impact on sFlt-1:PIGF ratio test in comparison with the current practice. A reduction in overall healthcare costs by $28 \%$ was observed. In particular, test implementation reduced $69.5 \%$ of unnecessary hospitalizations [22]. A similar cost-effectiveness study of PE testing in UK was published. The use of sFlt-1:PIGF ratio test translated into saving $£ 344$ per a pregnant woman [23]. NICE has been recommending using sFIt-1:PIGF ratio in the management of women with symptoms of PE since 2016 [24]. The test should be used to rule out PE in women with suspected PE between 20 and $34+6$ weeks of gestation.

Nowadays further studies on biochemical markers of $P E$ and their usefulness in algorithms of PE prediction are conducted. A new model of prediction of delivery with PE within one week in singleton pregnancies from 24 weeks to $36+6$ weeks of gestation in patients with suspected PE has been recently developed. It included sFlt-1/PIGF ratio, NT-proBNP and the gestational week at the time of the measurement. The addition of NT-proBNP improved the short-term prediction of delivery due to PE compared to sFlt-1/PIGF ratio alone [25]. Further prospective studies on a large population are necessary to estimate which biomarkers of PE have the highest utility in PE prediction.

\section{CONCLUSIONS}

PE remains one of the most important causes of maternal and perinatal mortality and morbidity. It is well established that ASA administered at a dose of $>100 \mathrm{mg}$ before 16 weeks of pregnancy reduces the risk of PE in a high risk population. Therefore, it is essential to identify women at risk of PE during the first trimester of pregnancy. Several algorithms for PE risk assessment have been developed and are available online. They include maternal factors combined with UTPI, MAP, PAPP-A, and PIGF expressed as multiples of the median values. Beside PE prophylaxis with ASA, a proper management of women considered to be at a high risk of $P E$ is essential. sFlt-1:PIGF ratio between 20 and $34+6$ weeks may be used to predict the short-term absence of PE in women in whom PE is clinically suspected or to predict the risk of PE diagnosis within 4 weeks and a significant shortening of duration of pregnancy associated with it. sFIt-1:PIGF ratio may be helpful in deciding about hospitalization or choosing the optimal time for corticosteroid administration in women at risk of PE. It may also help to reduce overall healthcare costs. 


\section{Acknowledgements}

None.

\section{Conflict of interest}

Nothing to declare.

\section{REFERENCES}

1. American College of Obstetricians and Gynecologists, Task Force on Hypertension in Pregnancy. Hypertension in pregnancy. Report of the American College of Obstetricians and Gynecologists'Task Force on Hypertension in Pregnancy. Obstet Gynecol. 2013; 122(5): 1122-1131, doi: 10.1097/01.AOG.0000437382.03963.88, indexed in Pubmed: 24150027.

2. Gallo DM, Wright D, Casanova C, et al. Competing risks model in screening for preeclampsia by maternal factors and biomarkers at 19-24 weeks' gestation. Am J Obstet Gynecol. 2016; 214(5): 619.e1-619.e17, doi: 10.1016/j.ajog.2015.11.016, indexed in Pubmed: 26627730.

3. Agrawal S, Cerdeira AS, Redman C, et al. Meta-Analysis and Systematic Review to Assess the Role of Soluble FMS-Like Tyrosine Kinase-1 and Placenta Growth Factor Ratio in Prediction of Preeclampsia:The SaPPPhirE Study. Hypertension. 2018; 71(2): 306-316, doi: 10.1161/HYPERTENSIONAHA.117.10182, indexed in Pubmed: 29229743.

4. The World Health Report 2005 - make every mother and child count. Geneva. World Health Organization.; 2005.

5. Ananth CV, Keyes KM, Wapner RJ. Pre-eclampsia rates in the United States, 1980-2010: age-period-cohort analysis. BMJ. 2013; 347: f6564, doi: 10.1136/bmj.f6564, indexed in Pubmed: 24201165.

6. Rolnik DL, Wright D, Poon LC, et al. Aspirin versus Placebo in Pregnancies at High Risk for Preterm Preeclampsia. N Engl J Med. 2017; 377(7): 613-622, doi: 10.1056/NEJMoa1704559, indexed in Pubmed: 28657417.

7. Roberge S, Bujold E, Nicolaides KH. Aspirin for the prevention of preterm and term preeclampsia: systematic review and metaanalysis. Am J Obstet Gynecol. 2018; 218(3): 287-293.e1, doi: 10.1016/j.ajog.2017.11.561, indexed in Pubmed: 29138036.

8. Regulation of the Minister of Health on the standards of medical proceedings when providing health care in obstetrics and gynaecology regarding perinatal obstetric and gynaecological care provided to women during pregnancy, labour and postpartum period, in cases of specific complications and care of women in cases of adverse pregnancy outcomes (as of 9 November 2015, Journal of Acts 2015, item 2007).

9. Kosinski P, Sarzynska-Nowacka U, Fiolna M, et al. The practical use of acetylsalicylic acid in the era of the ASPRE trial. Update and literature review. Ginekol Pol. 2018; 89(2): 107-111, doi: 10.5603/GP.a2018.0018, indexed in Pubmed: 29512816

10. The National Institute for Health and Care Excellence. Hypertension in pregnancy: diagnosis and management. Clinical Guidelines CG2017. London, UK: NICE.; 2011.

11. Wright $D$, Syngelaki $A$, Akolekar $R$, et al. Competing risks model in screening for preeclampsia by maternal characteristics and medical history. Am J Obstet Gynecol. 2015; 213(1): 62.e1-62.10, doi: 10.1016/j. ajog.2015.02.018, indexed in Pubmed: 25724400.

12. O'Gorman N, Wright $D$, Syngelaki $A$, et al. Competing risks model in screening for preeclampsia by maternal factors and biomarkers at 11-13 weeks gestation. Am J Obstet Gynecol. 2016;214(1): 103.e1-103.e12, doi: 10.1016/j.ajog.2015.08.034, indexed in Pubmed: 26297382.

13. Maynard SE, Min JY, Merchan J, et al. Excess placental soluble fms-like tyrosine kinase 1 (sFlt1) may contribute to endothelial dysfunction, hypertension, and proteinuria in preeclampsia. J Clin Invest. 2003; 111(5): 649-658, doi: 10.1172/JCl17189, indexed in Pubmed: 12618519.

14. Levine RJ, Lam C, Qian C, et al. CPEP Study Group. Soluble endoglin and other circulating antiangiogenic factors in preeclampsia. N Engl J Med. 2006; 355(10): 992-1005, doi: 10.1056/NEJMoa055352, indexed in Pubmed: 16957146.

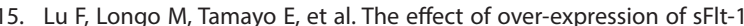
on blood pressure and the occurrence of other manifestations of preeclampsia in unrestrained conscious pregnant mice. Am J Obstet Gynecol. 2007; 196(4): 396.e1-7; discussion 396.e7, doi: 10.1016/j. ajog.2006.12.024, indexed in Pubmed: 17403433.

16. Levine RJ, Maynard SE, Qian C, et al. Circulating angiogenic factors and the risk of preeclampsia. N Engl J Med. 2004; 350(7): 672-683, doi: 10.1056/NEJMoa031884, indexed in Pubmed: 14764923.

17. Tsiakkas A, Saiid $Y$, Wright $A$, et al. Competing risks model in screening for preeclampsia by maternal factors and biomarkers at 30-34 weeks' gestation. Am J Obstet Gynecol. 2016; 215(1): 87.e1-87.e17, doi: 10.1016/j. ajog.2016.02.016, indexed in Pubmed: 26875953.

18. Andrietti $S$, Silva $M$, Wright $A$, et al. Competing-risks model in screening for pre-eclampsia by maternal factors and biomarkers at 35-37 weeks' gestation. Ultrasound Obstet Gynecol. 2016; 48(1): 72-79, doi: 10.1002/uog.15812, indexed in Pubmed: 26566592.

19. Zeisler H, Llurba E, Chantraine F, et al. Predictive Value of the sFlt-1:PIGF Ratio in Women with Suspected Preeclampsia. N Engl J Med. 2016; 374(1): 13-22, doi: 10.1056/NEJMoa1414838, indexed in Pubmed: 26735990.

20. Zeisler H, Llurba E, Chantraine F, et al. Soluble fms-Like Tyrosine Kinase-1-to-Placental Growth Factor Ratio and Time to Delivery in Women With Suspected Preeclampsia. Obstet Gynecol. 2016; 128(2): 261-269, doi: 10.1097/AOG.0000000000001525, indexed in Pubmed: 27399996.

21. Lapaire O, Shennan A, Stepan H. The preeclampsia biomarkers soluble fms-like tyrosine kinase-1 and placental growth factor: current knowledge, clinical implications and future application. Eur J Obstet Gynecol Reprod Biol. 2010; 151(2): 122-129, doi: 10.1016/j.ejogrb.2010.04.009, indexed in Pubmed: 20457483.

22. Frusca T, Gervasi MT, Paolini D, et al. Budget impact analysis of sFlt$1 /$ PIGF ratio as prediction test in Italian women with suspected preeclampsia. J Matern Fetal Neonatal Med. 2017; 30(18): 2166-2173, doi: 10.1080/14767058.2016.1242122, indexed in Pubmed: 27737599.

23. Vatish $M$, Strunz-McKendry $T$, Hund $M$, et al. sFlt-1/PIGF ratio test for pre-eclampsia: an economic assessment for the UK. Ultrasound Obstet Gynecol. 2016; 48(6): 765-771, doi: 10.1002/uog.15997, indexed in Pubmed: 27300726.

24. National Institute for Clinical Excellence. PIGF-Based Testing to Help Diagnose Suspected Pre-eclampsia (Triage PIGF Test, Elecsys Immunoassay sFIt-1/PIGF Ratio, DELFIA Xpress PIGF 1-2-3 Test, and BRAHMS sFlt-1 Kryptor/BRAHMS PIGF Plus Kryptor PE Ratio).

25. Sabrià $B a$, Lequerica-Fernández $P$, Lafuente-Ganuza $P$, et al. Addition of NT-proBNP to sFlt-1/PIGF ratio improves prediction of pre-eclampsia requiring delivery within 1 week, when sFlt-1/PIGF ratio is above 38: results of longitudinal cohort study. Ultrasound Obstet Gynecol. 2018 Mar 2.: Mar, doi: 10.1002/uog.19040. 